\title{
POLICY A research and policy agenda for the post-pandemic world
}

\author{
Authors: Chris Yiu, ${ }^{A}$ Benedict Macon-Cooney ${ }^{B}$ and Henry Fingerhut ${ }^{C}$
}

The COVID-19 pandemic response has engaged the academic, public, private and health sectors in the real-time development of technologies and practices to enable predictive, preventive, personalised and participatory (P4) health. Myriad cases of collaborative innovation across these sectors have emerged throughout the pandemic response (despite certain observed technical, social and institutional barriers) that serve as examples to address post-pandemic health system challenges. In this paper, we propose a joint research and policy agenda to generate the knowledge and practices to identify and extend these acute gains toward chronic health system challenges in the post-pandemic era. We identify three key themes for postpandemic research and policy: the dialectic between novel and traditional techniques, the tension between centralised and local decision-making, and cooperation across academic disciplines, sectors and borders. Going forward, attention to these three themes by researchers and policymakers will help align our health, policy, academic and technological systems to provide better health for all.

KEYWORDS: health systems, health policy, COVID-19, healthcare delivery, innovation policy

DOI: $10.7861 /$ fhj.2021-0082

\section{Introduction}

The technological revolution of the 21st century is re-orientating the world. New paradigms are being created, with new risks and new opportunities, with consequences that will be far greater in depth than the industrial revolution of the 20th century. The COVID-19 pandemic response has demonstrated the opportunities but also the technical, social, and institutional challenges of developing and applying predictive, preventive, personalised and participatory (P4) health technologies, harnessing this revolution to work for all on a rapid timescale that

Authors: A executive director of technology and public policy, Tony Blair Institute for Global Change, London, UK; ${ }^{B}$ director of science \& innovation unit, Tony Blair Institute for Global Change,

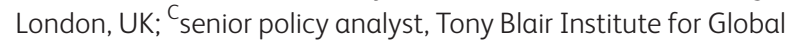
Change, London, UK corresponds to that of the challenges we face. ${ }^{1,2}$ Some of the shape of the future economy has been revealed by the current pandemic: internet era companies continued to thrive as much of the world around them faltered. And in health, the potential of breakthroughs in biotech have been laid out before us in the most profound way. Many lives will ultimately be saved by the speed at which vaccines were developed.

But as nations confront the task ahead, there is a clear need to go faster in supporting the application of technology to address our most pressing health challenges. ${ }^{3}$ The need for policymakers to invest in networked technological infrastructure for healthcare provision, take a platform approach to support decentralised health innovators and build trust in emerging health technologies to support their adoption is more urgent than ever.

A little more than a year since the onset of the pandemic, advances have been made in all three domains that would not have been politically or culturally possible in 2019. But the unequal access to services and vaccines at the local, national and global scales, and the sheer scale of illness and mortality demonstrate just how far we have yet to go.

Three related themes to support health technologies have also emerged for the management of health research priorities and the application of research to policy more broadly as we emerge from the pandemic (Table 1). Where Blair and Yiu identify the challenge of trust in emerging health technologies, the pandemic has raised more broadly the dialectic between novel and traditional techniques. ${ }^{3}$ The pandemic has demonstrated the tension between centralised and local decision making, to which, the authors' platform approach to decentralised innovation responds. In addition to the challenge of coordinating between levels, the pandemic has identified the need for cooperation across academic disciplines, sectors and borders, to which, the authors' call for networked technological infrastructure responds.

Here, we present successes and ongoing challenges from across three domains in which these themes have emerged during the pandemic: evidence generation and use in decision making, health system operations, and public health. This agenda is critical and, as our recent history has shown, will require a broad coalition. It requires us to engage researchers from across academic disciplines and policymakers from across public sector agencies and roles, as well as those working at the forefront of technology today. COVID-19 should be a collective wake-up call that we need deeper and more concerted action to improve the resilience of our health systems to future shocks. We must ensure that our health, 
Table 1. Opportunities and future challenges for generating and applying health research evidence to policy and practice

$\begin{array}{ll}\begin{array}{l}\text { Novel vs } \\ \text { existing }\end{array} & \text { Use of non-traditional data sources (search } \\ \text { techniques } & \text { queries, Twitter sentiment, smartphone } \\ & \text { mobility or wearables). } \\ & \text { Predictive, preventive, personalised and } \\ & \text { participatory (P4) precision medicine. } \\ & \text { Digital contact tracing. } \\ \text { Centralised vs } & >\text { Coordination challenges among research } \\ \text { local decision } & \text { groups. } \\ \text { making } & >\text { Incentives, mechanisms and structures for } \\ & \text { largescale open data sharing. } \\ \text { Cooperation } & \text { Knowledge management and preprints. } \\ \text { across } & \text { Ad hoc interdisciplinary research } \\ \text { disciplines, } & \text { collaborations (eg COVID19 Dispersed } \\ \text { sectors, and } & \text { Volunteer Research Network). } \\ \text { borders } & \text { Rapid funding mechanisms. } \\ & \text { Data journalism, public communication } \\ & \text { and transparency. } \\ & \text { Government science advisory committees. } \\ & \text { Realtime evaluation and collaboration } \\ & \text { among hard scientists, ethicists and } \\ & \text { economists to inform human challenge } \\ & \text { trial debate; UK Human Challenge } \\ & \text { Consortium. }\end{array}$

policy, academic and technological systems are aligned to provide better health for all.

\section{Novel vs established data, methods and techniques}

The COVID-19 pandemic required innovation from within the academic, public, private and health sector communities. In record speed, researchers developed new theory, methods and ways of working, and these three aspects interacted to produce new knowledge about biological and social systems and processes affecting disease dynamics. In near real time, researchers sequenced the coronavirus genome, developed tests and validated vaccines via novel mechanisms including the novel mRNA platform and the vaccine platform mechanism more broadly. ${ }^{4}$ In terms of methods, time dynamics of testing required the use of novel data sources and valid methods for including them in both policymaking and academic theory development. ${ }^{5,6}$ Novel datasets and new testing have been essential to identifying emergent trends quickly, informing both theory generation and policy, and enabling governments to reduce a 5 -week decision cycle imposed by disease and testing dynamics. These advances were made while most researchers adapted to remote work in response to the pandemic, opening new opportunities for interaction and collaboration across institutions and borders. ${ }^{\text {? }}$

Novel techniques nevertheless present challenges from both ethical and validity standpoints. Leslie offers five steps for responsible data use and privacy-preserving innovation to respond to the COVID-19 pandemic; these steps fit more broadly into a research agenda going forward, providing a third way between the false choice of compromising privacy on the one hand and pandemic response on the other. ${ }^{8}$ From a validity standpoint, novel mechanisms such as the use of non-traditional data sources for near real-time epidemiological modelling also have their limitations, one notable example being the use of Google search queries for flu prediction. ${ }^{9}$ It is essential to maintain evidence standards for both theory generation and policymaking purposes, not by dismissing these techniques out of hand but by continuing to refine research methods such that they can be used responsibly within a suite of indicators to provide valid, near real-time insights. Anticipatory innovation models provide a structured process to consider the technical, biological, temporal, spatial and political uncertainties and possible futures that may arise and to systematise the technical, political and knowledge management mechanisms that have enabled digital health innovation at speed that have emerged during the pandemic. These approaches, bringing together technologists, interdisciplinary academics, policymakers and citizens, are increasingly important to engage digital transformation toward democratic ends. ${ }^{10}$

\section{Coordination between levels: centralised vs local decision making}

Across fields, hierarchical top-down/bottom-up models have given way to networked organisations. Indeed, the pandemic has demonstrated the longstanding tension between centralised and local decision making; administrations juggled the coordination and standardisation benefits of centralised decision making with the need for agility, adaptation to local conditions and experimentation that decentralised decision making brings, to varying degrees of success. ${ }^{11,12}$ The UK government has laid out guiding principles for local authorities in the interest of a coordinated set of decisionmaking criteria across levels of government. ${ }^{13}$

In the academic sphere, research coordination quickly became an issue; researchers moved quickly to adapt their research agendas to address the pandemic but, in doing so, left important gaps, such as the study of COVID-19 in children. ${ }^{14}$ Further, the commendable rush to contribute to pressing COVID-19 research nevertheless threatened to slow important, existing research in other areas of public health. ${ }^{15}$ In the public sector, countries moved quickly to establish the data infrastructure necessary to aggregate disparate epidemiological, health systems and economic data; the different approaches they have taken to data collection, user journeys that generate data, organisations involved and aggregation, and analysis will indicate best practices going forward for the aggregation and use of disparate, real-time data in health policy. ${ }^{16,17}$ The design and use of intersectoral data systems contributes to the 'situational awareness' necessary for the precision policy decisions that represent a third way between overly permissive policies that allow the virus to propagate and burden health systems and overly restrictive policies that burden the economy and constrain civil liberties. ${ }^{18}$

Funding mechanisms like the EU Coronavirus Pledging Conference created necessary support and coordinating signals that helped align the disparate international research groups contributing to COVID-19 research to ensure research was allocated to necessary areas. ${ }^{19}$ Data portals similarly enabled researchers to centralise data to enable open science and rapid advancement of the knowledge generation and peer review processes. ${ }^{20}$ Whole-genome sequences were available to international researchers on GISAID as early as January 2020 and 
the open access it provides to over 450,000 genome sequences was essential to vaccine development; similarly, the COVID-19 Genomics UK Consortium centralises over 370,000 sequences identified through UK testing programmes and coordinates genomic analysis contributing to regular reports to SAGE on virus mutation. ${ }^{21,22}$

\section{Coordination across organisations, disciplines, sectors and borders}

Academics have greatly expanded the use of new mechanisms of collaboration across organisations and disciplines, as well as communicating research to policymakers and the public. Researchers developed ad hoc research collaborations across disciplines, institutions and borders (such as the COVID-19 Distributed Volunteer Research Network) to quickly develop crosscutting research that addressed the biological, epidemiological, legal and ethical aspects of disease dynamics and interventions. ${ }^{7}$

Mechanisms, such as preprints, fast-track publishing and scientific advisory committees (eg the UK government's SAGE), also acted as portals for policymakers and journalists to quickly acquire and act on knowledge from the academic sphere. But while preprints enabled academics to collaborate and policymakers and journalists to track research findings in real time, these external communities were not necessarily equipped to understand the theoretical context and communication for an academic audience, leading to calls for strategies to govern the use of preprints, particularly as scientific debate has played out in the public in real time. ${ }^{23-25}$

An interdisciplinary response is essential for this pandemic, in which macro policy choices were quickly framed as a trade-off between economic and health outcomes, ethical considerations of privacy and equity have come to the fore, and individual behaviour is paramount to disease dynamics. The UK government's Scientific Pandemic Insights Group on Behaviours (SPI-B) designed principles for co-production of guidance between policymakers and governed populations to equitably establish local behavioural standards and policies, increasing acceptability and effectiveness of resulting policies. ${ }^{26}$ The question of vaccine challenge trials brought together researchers, health system administrators, private companies and governments to consider both the scientific and ethical merits, resulting in the approval of the Human Challenge Programme in the UK. ${ }^{27}$ We would argue that a joint letter from leading researchers (including virologists, epidemiologists, economists, philosophers and ethicists) actually represents a promising achievement in its own right, as a rapid, interdisciplinary policy proposal that unites expert opinion on the scientific and ethical concerns. ${ }^{28}$

However, these policy trade-offs are particularly challenging because, despite admonitions to 'follow the science', under evidence-based policymaking, policymakers trade off policy priorities (an inherently value-driven and political process that depends on scientist navigation of the policy process) informed by available evidence. ${ }^{29,30}$ However, the interdisciplinary nature of these decisions means that they are also implicitly trading off silos of academic knowledge and disparate groups of scientific advisors with different expertise and norms.

Similarly, as public compliance with preventive behavioural measures is essential to mitigating this respiratory pandemic, public understanding of and confidence in scientific findings is a critical component of pandemic response. Innovative public communication and transparency tools (such as the GOV.UK dashboard) and novel approaches to data journalism were developed in short order to communicate the breadth of relevant epidemiological and operational data and visualisations interactively, in real time, and at scales that enable individuals to both hold government to account and make informed behavioural decisions. ${ }^{31,32}$ As the public engages with these resources, effects such as 7-day moving averages, test sensitivity/specificity, timeseries lags and demographic or geographic multimodality are routinely covered, data literacy, numeracy and susceptibility to cognitive biases are likely to change. The pandemic will not itself resolve a trend of low numeracy in the general public, but advances in data journalism over the past year have gone a long way toward integrating these concepts into common policy discourse. ${ }^{33}$ Scientists themselves have also taken to social media to establish collaborations with a wider sphere of academics and to engage directly with the public on the scientific process and their own field, correcting popular misperceptions (or deliberate misrepresentations) of the scientific record and narrowing the gap between the academic and public spheres.

Academic institutions have increasingly partnered with private sector and healthcare organisations to improve translation of their research and inform the design of promising innovations. Collaborations like the new partnership between the P4 Precision Medicine Accelerator (an ecosystem for precision medicine startups) with Nuffield Health bring together private start-ups, healthcare organisations and academics to co-design and test promising innovations, improving their relevance to practice and reducing time to development. ${ }^{34}$ These initiatives, along with the advancement of the implementation science field, help systematise the design of practice-relevant technologies and their adoption across settings to close the implementation gap and improve health service quality. ${ }^{35}$

At the international level, coordination across countries and regions is a key example of the limits of centralised decisionmaking, as multilateral institutions rely on coordination among member states in the absence of hard power. While multilateral institutions, notably the World Health Organization, had a coordinated pandemic response plan in place, they had few mechanisms or hard power to compel nations to act together. ${ }^{36}$ Most recently, the EU vaccine distribution strategy further demonstrated the challenges of achieving international consensus to vet, procure and distribute specific vaccines. ${ }^{37}$ While COVID-19 drew exceptional attention because it impacted the world's population, the restrictions on mobility have also impeded efforts to address continuing global health and sustainable development challenges that disproportionately or exclusively impact low- and middle-income countries, doubly impacting these populations. ${ }^{38}$

\section{Recommendations: $a$ joint research and policy agenda}

As Blair and Yiu argue, in setting the agenda for future health policy: 'the specific technologies matter less than the shift in mindsets and approaches that the modern operating environment makes possible. ${ }^{3}$ We recommend a cross-cutting shift in mindsets and approaches that will strengthen our capacity for health research and its relevance for public policy. The pandemic has laid bare the challenges and opportunities in applying research 
to policy, and a concerted effort across academia and the public sector is necessary to create the structures, mechanisms and norms for effective evidence-based policymaking.

To this end, we propose the following recommendations to shape global health research policy over the immediate, medium and long term, including target outcomes and illustrative initiatives to achieve them.

\section{Mechanisms to support interdisciplinary, problem-} focused research

Interdisciplinary research has been gradually increasing and is particularly prominent in the health disciplines, however interdisciplinary studies take longer to gain traction. ${ }^{39}$ But true interdisciplinary research, effectively melding traditional disciplines to create knowledge that would not have been possible otherwise because it spans the boundary assumptions of separate disciplines, takes hard intellectual work, investment in a shared mission and engagement norms, and is under-incentivised by traditional academic norms, institutional structures and likelihood of funding. ${ }^{40,41}$ Government funders, donors, academic institutions and learned societies should create incentives for the conduct of interdisciplinary research that support both problem-oriented findings and the development of systematic research methods to combine cross-disciplinary approaches.

> Formal training in interdisciplinary methods for PhD students.

$>$ Research prizes to honour problem-focused interdisciplinary achievements.

> Experimental funding mechanisms to support ambitious interdisciplinary research and incentivise collaboration across disciplines, building on the USA's DARPA and UK's ARIA models.

\section{Mechanisms to improve co-creation of applied research / policy agendas}

Most scientists are untrained in the policy process and focus on packaging and communicating evidence based on scientific standards, resulting in a form of information that may not be relevant for policymakers. ${ }^{30}$ Further, scientists are disincentivised from contributing applied, policy-relevant analysis within the policy sphere due to competitive funding, publishing and tenure mechanisms that value teaching, research and academic service contributions. In one study, academics were particularly attentive to incentives to provide monetary support, professional recognition, academic promotion and capacity enhancement, particularly relevant to expand access to science advice beyond the majority white, male and, in the UK, London-based 'usual suspects' ${ }^{\text {42,43 }}$

> Governments should consider creating an 'academic reserve,' a standing funding mechanism for academics to contribute applied analysis to support public service design and policy.

> Universities should develop public policy units (eg University College London's Science, Technology, Engineering and Public Policy department and Massachusetts Institute of Technology's Joint Program on the Science and Policy of Global Change) to provide a point of entry to facilitate policy advice based on scientists' research and to educate scientists on the policy process.

> Governments should extend the use of expert groups in government (eg SAGE, SPI-B and HDR UK) beyond crisis periods and expand access to these groups to include diverse academics across disciplines, institutions, personal backgrounds and geographic settings. They should also add experts in public policy, evidence-based policymaking and ethics to scientific advisory groups to facilitate the application of science within the policy process.

\section{A strategy for science communications}

Despite advances by scientists, journalists and government agencies in data communication methods throughout the pandemic, statistical and data literacy is increasingly important to engage the public in response to pressing health policy challenges. Individuals and organisations at the local, national and international levels have developed myriad resources for educating the general public in the basic statistical literacy and numeracy skills necessary to interpret data that impacts their health. ${ }^{44-46}$ Going forward, it is more important than ever to coordinate and adapt these resources to the local cultural and educational contexts (through both formal curricula and informal general communication campaigns) to improve awareness and help students, journalists and the general public develop statistical comprehension skills in a meaningful and engaging way.

> Individual scientists should expand their efforts to communicate their own research and the current state of their field on social media in order to engage other academics across disciplines and the general public.

> Scientists, journalists and civil servants should continue to experiment to devise novel communication techniques to improve scientific, statistical and data literacy among policymakers and the general public.

$>$ Public sector, academic and media institutions should collaborate to design a numeracy and statistical literacy education campaign for children and adults. This campaign should focus on novel, light-touch and age-appropriate approaches to engage the general public in everyday statistical thinking, complementing dedicated science, technology, engineering and mathematics (STEM) curricula in schools and universities.

\section{Health technology development, implementation and evaluation}

While the pandemic has accelerated progress toward the health technology agenda proposed by Blair and Yiu, more research and public investment will be necessary to evaluate these advances, advise on their use in non-crisis times and adapt health technologies to ensure they work for all.

> Researchers should partner with health systems to conduct a ground-up, post-pandemic technology audit, in order to identify critical health technology infrastructure and innovations that should be integrated into national health systems going forward. Technologies should be used equitably and responsibly to ensure they improve health outcomes for all.

> Academic institutions should expand translational research initiatives (including collaborations with private companies, accelerators and healthcare systems) to develop promising precision and predictive health technologies and complement these initiatives with implementation research programmes to enable uptake at scale. 
These recommendations are not intended to replace existing research and policy approaches, it is essential to maintain basic and applied research that is rightly independent of government's policy priorities for the purposes of knowledge creation, horizon setting and holding policymakers to account. Rather, these recommendations are intended to align those research initiatives meant to align with policy priorities to be conducted to greatest effect, and to provide indications as to where the research enterprise can be expanded.

\section{Conclusion}

As we emerge from this global public health challenge, it is more important than ever for academics and policymakers to work together to evaluate and improve our health systems and policy. We should draw lessons and inspiration from innovative successes in knowledge generation, policymaking, technology development and ways of working, while acknowledging the magnitude of loss and unequal outcomes across communities and working to resolve limitations in preparedness, system design and policy that have contributed to those adverse outcomes.

Now more than ever, the health research, policy and operations communities are in the spotlight for the critical work they have done to stem this global pandemic. Working together across disciplines, sectors and borders, we will be well-positioned both to protect against future shocks and to improve long-term health outcomes for all.

\section{References}

1 Hood L, Balling R, Auffray C. Revolutionizing medicine in the 21st century through systems approaches. Biotechnology Journal 2012; 7:992-1001.

2 Macon-Cooney B. Revolutionising health: a human grand challenge. Institute for Global Change, 2020. https://institute.global/ policy/revolutionising-health-human-grand-challenge

3 Blair T, Yiu C. Challenges and opportunities for future health policy. Future Healthcare Journal 2019:6:88-9.

4 van Riel D, de Wit E. Next-generation vaccine platforms for COVID19. Nature Materials 2020;19:810-2.

5 Majumder MS, Santillana M, Mekaru SR et al. Utilizing nontraditional data sources for near real-time estimation of transmission dynamics during the 2015-2016 Colombian Zika virus disease outbreak. JMIR Public Health Surveill 2016;2:e30.

6 Santillana M, Nguyen AT, Dredze M et al. Combining search, social media, and traditional data sources to improve influenza surveillance. PLOS Computational Biology 2015;11:e1004513.

7 Majumder MS. Coronavirus researchers are dismantling science's ivory tower-one study at a time. Wired, 2020. www.wired.com/ story/covid-19-studies-dismantle-science-ivory-tower

8 Leslie D. Tackling COVID-19 through responsible AI innovation: five steps in the right direction. Harvard Data Science Review, 2020. https://hdsr.mitpress.mit.edu/pub/as1p81um

9 Lazer D, Kennedy R, King G, Vespignani A. The parable of Google Flu: traps in big data analysis. Science 2014;343:1203-5.

10 Bayram M, Springer S, Garvey CK, Özdemir V. COVID-19 digital health innovation policy: a portal to alternative futures in the making. OMICS: A Journal of Integrative Biology 2020:24:460-9.

11 Berman $E$. The roles of the state and federal governments in a pandemic. Journal of National Security Law and Policy 2020;11:61-82. https://jnslp.com/wp-content/uploads/2020/12/The-Roles-of-theState-and-Federal-Governments-in-a-Pandemic_2.pdf

12 Malandrino A, Demichelis E. Conflict in decision making and variation in public administration outcomes in Italy during the COVID-19 crisis. European Policy Analysis 2020;6:138-46.
13 Department of Health and Social Care. COVID-19 contain framework: a guide for local decision-makers. DHSC, 2021. www.gov.uk/ government/publications/containing-and-managing-localcoronavirus-covid-19-outbreaks/covid-19-contain-framework-aguide-for-local-decision-makers

14 Bourgeois FT, Avillach P, Turner MA. The urgent need for research coordination to advance knowledge on COVID-19 in children. Pediatr Res 2020 [Epub ahead of print].

15 Pletschette M, Lienhardt C, Brigden G et al. COVID-19 research in Europe needs coordination, but we must not stop European research investments in poverty related diseases. BMJ Opinion 2020. https://blogs.bmj.com/bmj/2020/08/24/covid-19-researchin-europe-needs-coordination-but-we-must-not-stop-europeanresearch-investments-in-poverty-related-diseases/

16 Dace $\mathrm{H}$. The missing gaps in COVID-19 data: reflections from seven country case studies. Institute for Global Change, 2021. https://institute.global/policy/missing-gaps-covid-19-datareflections-seven-country-case-studies

17 Fingerhut $\mathrm{H}$. Building real-time data infrastructure into the heart of public health. Tony Blair Institute for Global Change, 2021. https:// institute.global/policy/building-real-time-data-infrastructure-heartpublic-health

18 Bamford R, Dace H, Macon-Cooney B, Yiu C. A price worth paying: tech, privacy and the fight against COVID-19. Institute for Global Change, 2020. https://institute.global/policy/price-worth-payingtech-privacy-and-fight-against-covid-19

19 European Union. Coronavirus global response. European Union, 2020. https://global-response.europa.eu/index_en

20 EMBL-EBI. Open data sharing accelerates COVID-19 research. EMBL-EBI, 2020.

21 van Noorden R. Scientists call for fully open sharing of coronavirus genome data. Nature 2021;590:195-6.

22 COVID-19 Genomics UK Consortium. COVID-19 Genomics UK Consortium. COG-UK, 2020. www.cogconsortium.uk

23 Majumder MS, Mandl KD. Early in the epidemic: impact of preprints on global discourse about COVID-19 transmissibility. Lancet Global Health 2020;8:e627-30.

24 Brierley L. Lessons from the influx of preprints during the early COVID-19 pandemic. Lancet Planetary Health 2021;5:e115-7.

25 BMJ talk medicine. What should 'following the science' mean for government policy? BMJ talk medicine, 2021. https://soundcloud. com/bmjpodcasts/what-should-following-the-science-mean-forgovernment-policy

26 Bear L, Melendez-Torres G], Solanke I. SPI-B: Principles for co-production of guidance relating to the control of COVID-19, 8 July 2020. Scientific Advisory Group for Emergencies, 2020. www.gov.uk/government/ publications/spi-b-principles-for-co-production-of-guidance-relating-tothe-control-of-covid-19-8-july-2020

27 Kirby T. COVID-19 human challenge studies in the UK. Lancet Respir Med 2020;8:e96.

28 Aaronson S, Ahuja A, Anderson C et al. UK: Challenge trials for COVID-19. 1 Day Sooner, 2020. www.1daysooner.org/uk-open-letter

29 Stevens A. Governments cannot just 'follow the science' on COVID19. Nat Hum Behav 2020;4:560.

30 Cairney P, Oliver K. Evidence-based policymaking is not like evidencebased medicine, so how far should you go to bridge the divide between evidence and policy? Health Research Policy and Systems 2017:15:35.

31 UK Government. Coronavirus in the UK: Daily summary. GOV.UK 2020. https://coronavirus.data.gov.uk

32 The New York Times. IRE honors for coronavirus tracking project. The New York Times 2021. www.nytco.com/press/i-r-e-honors-forcoronavirus-tracking-project

33 de Langhe B. COVID-19 vaccine trials are a case study on the challenges of data literacy. Harvard Business Review 2020. https:// hbr.org/2020/12/covid-19-vaccine-trials-are-a-case-study-on-thechallenges-of-data-literacy 
34 Nuffield Health. Partnership with P4 precision medicine accelerator. Nuffield Health, 2020. www.nuffieldhealth.com/article/partnershipwith-p4-precision-medicine-accelerator-to-fast-track-innovation-inprecision-medicine

35 Eccles MP, Mittman BS. Welcome to Implementation Science. Implementation Science 2006;1:1.

36 Patrick S. When the system fails: COVID-19 and the costs of global dysfunction. Foreign Affairs, 2020. www.foreignaffairs.com/articles/ world/2020-06-09/when-system-fails

37 BBC News. COVID: EU's von der Leyen admits vaccine rollout failures. BBC News, 2021. www.bbc.co.uk/news/world-europe-56009251

38 Lambert H, Gupte J, Fletcher H et al. COVID-19 as a global challenge: towards an inclusive and sustainable future. Lancet Planet Health 2020:4:e312-4.

39 van Noorden R. Interdisciplinary research by the numbers. Nature 2015;525:306-7.

40 Brown RR, Deletic A, Wong THF. Interdisciplinarity: How to catalyse collaboration. Nature 2015;525:315-7.

41 Bromham L, Dinnage R, Hua X. Interdisciplinary research has consistently lower funding success. Nature 2016;534:684-7.

42 Jessani NS, Valmeekanathan A, Babcock CM, Ling B. Academic incentives for enhancing faculty engagement with decisionmakers-considerations and recommendations from one School of Public Health. Humanities and Social Sciences Communications 2020;7:148.

43 Sasse T. Academics need support to increase their policy impact. Institute for Government, 2019. www.instituteforgovernment.org. uk/blog/academics-need-support-increase-their-policy-impact

44 United Nations Economic Commission for Europe. Making data meaningful: Part 4: How to improve statistical literacy: A guide for statistical organizations. United Nations, 2014. https://unece.org/ statistics/making-data-meaningful

45 Sanchez J (ed). Government Statistical Offices and Statistical Literacy. International Statistical Literacy Project, 2008. https:// iase-web.org/islp/Publications.php?p=Books

46 Royal Statistical Society. Improve your statistical literacy. Royal Statistical Society. https://rss.org.uk/training-events/training/ stats-resources/improve-your-statistical-literacy

Address for correspondence: Dr Henry Fingerhut, Tony Blair Institute for Global Change, One Bartholomew Close, London EC1A 7BL, UK.

Email: h.fingerhut@institute.global

Twitter: @HenryFingerhut 